\title{
25 Research Soure \\ Finding the "switch" in platelet activation Prediction of key mediators involved in platelet hyperreactivity using a novel network biology approach
}

TP Lemmens

Maastricht University

DM Coenen

University of Kentucky College of Medicine

ICL Niessen

Maastricht University

F Swieringa

Maastricht University

SLM Coort

Maastricht University

RR Koenen

Maastricht University

M Kutmon

Maastricht University

JMEM Cosemans ( $\sim$ judith.cosemans@maastrichtuniversity.nl )

Maastricht University

\section{Research Article}

Keywords: platelet activation and inhibition, data integration, platelet reactivity, molecular switch

Posted Date: August 24th, 2021

DOI: https://doi.org/10.21203/rs.3.rs-692247/v1

License: (a) This work is licensed under a Creative Commons Attribution 4.0 International License.

Read Full License

Version of Record: A version of this preprint was published at Journal of Proteomics on April 5th, 2022. See the published version at https://doi.org/10.1016/j.jprot.2022.104577. 


\section{Abstract}

The healthy endothelium controls platelet activity through release of prostaglandin I2 $\left(\mathrm{PGI}_{2}\right)$ and nitric oxide. The loss of this natural brake on platelet activity can cause platelets to become hyperreactive. $\mathrm{PGI}_{2}$ attenuates platelet activation by adenosine diphosphate (ADP) through stimulation of cyclic adenosine monophosphate (CAMP) production and subsequent phosphorylation changes by protein kinase A (PKA). We hypothesize that proteins/processes involved in platelet hyperactivity downstream of the cAMP-PKA pathway can serve as a "switch" in platelet activation and inhibition.

We designed a network biology approach to explore the entangled platelet signaling pathways downstream of $\mathrm{PGI}_{2}$ and ADP. The STRING database was used to build a protein-protein interaction network from proteins of interest in which we integrate a quantitative platelet proteome dataset with pathway information, relative RNA expression of hematopoietic cells, the likelihood of the proteins being phosphorylated by PKA, and drug-target information from DrugBank in a biological network.

We distilled 30 proteins from existing phosphoproteomics datasets (PXD000242 and PXD001189) that putatively can be "turned on" after ADP-mediated platelet activation and subsequently switched "off" after platelet inhibition with iloprost. Enrichment analysis revealed biological processes related to vesicle secretion and cytoskeletal reorganization to be overrepresented coinciding with topological clusters in the network. Our method highlights novel proteins related to vesicle transport, platelet shape change, and small GTPases as potential switch proteins in platelet activation and inhibition. Our novel approach demonstrates the benefit of data integration by combining tools and datasets and visualization to obtain a more complete picture of complex molecular mechanisms.

\section{Background}

Endothelial dysfunction is a common feature of cardiovascular diseases such as atherosclerosis and hypertension [1, 2]. It is characterized by reduced availability of nitric oxide (NO) and the inability to adequately maintain vascular homeostasis [3]. Normally, the endothelium limits platelet activity and aggregation through prostaglandin $\mathrm{I} 2\left(\mathrm{PGI}_{2}\right)$ and NO. The loss of this natural brake on platelet activity can cause platelets to become hyperreactive, which contributes to the progression of cardiovascular diseases [4]. For instance, insulin resistance and hypoglycemia in type 2 diabetes mellitus (T2DM) are known to lead to reduced platelet sensitivity to $\mathrm{PGI}_{2}$ and $\mathrm{NO}[5,6]$. Furthermore, platelet hyperreactivity can contribute to antiplatelet drug resistance, which is associated with poor cardiovascular outcome [79]. $\mathrm{PGI}_{2}$ attenuates platelet activation through binding to the IP platelet membrane receptor, inducing activation of adenylate cyclase (AC), and subsequent production of cyclic adenosine monophosphate (CAMP) and cAMP-dependent activation of protein kinase A (PKA). PKA phosphorylates a range of proteins, consequently leading to inhibition of platelet aggregation, secretion, and shape change. Activation of the Gi-coupled adenosine diphosphate (ADP)-P2Y ${ }_{12}$ receptor leads to inhibition of $A C$, thereby releasing the natural brake on platelet activation. A second main signaling route downstream of 
$P 2 Y_{12}$ is activation of phosphoinositide 3-kinase ( $\left.P I 3 K\right)$, which leads to activation of the platelet integrin $a_{11 b} \beta_{3}$ receptor and enables platelet aggregation [10]. Next to $P 2 Y_{12}$, ADP activates platelets via the Gqcoupled $\mathrm{P} 2 \mathrm{Y}_{1}$ receptor, which is essential for platelet shape change via phospholipase $\mathrm{C}$ stimulation [11]. Taken together, this highlights the cAMP-PKA signaling axis as an interesting starting point to identify novel key players in platelet activation and inhibition.

We hypothesize that downstream of the cAMP-PKA pathway proteins or processes are present that can serve as a "switch" in platelet activation and inhibition. Molecular switches are abundant in many different biological functions. A known molecular switch in platelet (in)activation is Rap1, a small GTPase that is essential in integrin $a_{\| b} \beta_{3}$ activation after stimulation of $G$ protein-coupled receptors (GPCR) [12]. Hydrolyzation of Rap1b-GTP by the GTPase Ras GTPase-activating protein 3 (RASA3) to a GDP-bound form causes the protein to be switched off [13]. Binding of ADP to P2Y ${ }_{12}$ results in inhibition of RASA3 by PI3K, thereby driving $a_{\| b} \beta_{3}$ activation and platelet aggregation [14]. Besides this known example of a switch protein, the process of platelet activation/hyperreactivity relies on many more proteins that form a complex system of pathways with numerous interactions. In this article we aim to find proteins that can be utilized as targets to reverse platelet hyperreactivity. We developed a network biology approach to integrate information from different data sources to address our aim and to explore the entangled platelet signaling pathways downstream of $\mathrm{PGI}_{2}$ and $\mathrm{ADP}$.

\section{Methods}

We designed a network biology approach to find putative switches in platelet activation and inhibition. The workflow that we developed is schematically represented in Fig. 1 and explained below.

Phosphoproteomics dataset

To create a selection of proteins with putative involvement in the resolution of platelet activation, we made use of publicly available phosphoproteomics datasets. Beck et al. reported on the phosphoproteomics response of platelets treated with iloprost (a stable prostacyclin analogue) for 10, 30 and 60 seconds (dataset containing 2739 phosphopeptides; 360 proteins with regulated (99\% confidence) phosphorylation) [16]. Additionally, they reported on a dataset combining iloprost (30 and 60 seconds) and ADP stimulation (30 seconds) on platelet protein phosphorylation (containing $>4797$ phosphopeptides; 608 proteins regulated) [15]. These datasets can be accessed through ProteomeXchange under the accession PXD000242 and PXD001189, respectively. In the present study we extracted proteins from these datasets based on known platelet signaling routes, see paragraph 'Data selection and characterization' under Results for detailed description.

Network generation

We utilized the StringApp [17] in Cytoscape 3.7.2 [18] to build a protein-protein interaction network with the 30 selected proteins involved in the resolution of platelet activation based on known pathway information downstream IP and $\mathrm{P}_{2} \mathrm{Y}_{12}$ as protein query. A confidence score cut-off of 0.5 was selected to 
include enough nodes in the network, while maintaining a high selectivity [17]. Subsequently, the network was expanded to incorporate an additional 20 interactors based on their total connectivity relative to their overall connectivity in the STRING database. These proteins may provide mechanistic links between nodes in the network and thereby might reveal relevant processes. All processes were automated in $\mathrm{R}$ (version 3.6.3) using the RCy3 package [19].

Functional enrichment analysis

Gene Ontology (GO) enrichment analysis on all proteins in the resulting network and their corresponding genes was performed using the integrated tool from PANTHER on the GO website (http://geneontology.org/) [20-22]. This was done to see which processes were important in relation to platelet activation and inhibition. Main settings that were used are: Biological process, Homo sapiens, Fisher's exact test type and false discovery rate as correction $(P<0.05)$. We used a default enrichment significance threshold of 0.05 to find significantly overrepresented pathways or processes corresponding to clusters observed in the network.

Protein Kinase A prediction and druggability

GPS 5.0 is a tool to predict and score phosphorylation sites based on their kinase consensus sequence [23]. A higher score indicates an increased probability for protein phosphorylation at the specific site by the specified protein kinase. The threshold was set to the highest probability and the outcome was visualized on nodes using Cytoscape. We used this tool to confirm that the proteins in our selection could be phosphorylated by PKA.

Information on FDA-approved drugs and their interactions from the DrugBank database version 5.1.0 [24] were incorporated into the network using CyTargetLinker [25] and visualized in Cytoscape. CyTargetLinker is a Cytoscape application integrating different "linksets", e.g., drug-target, microRNA-target, and regulatory interactions into a network.

Copy number and relative RNA expression

We incorporated existing quantitative data on the platelet proteome dataset in our network to show the estimated copy number in human platelets [26]. Although other factors play a role, this gives a good indication on the importance of a specific protein. To obtain an indication of the relative expression of proteins in our network compared to other hematopoietic cells, we used Blood RNAexpress, which is made freely available by the Blueprint consortium [27]. Even though RNAseq data and proteomic datasets do not correlate very well in platelets. It can still give an indication as to what proteins are worth investigating further.

\section{Results}

Data selection and characterization 
Knowing that the cAMP-PKA pathway is a major platelet inhibitory pathway [28] and that phosphorylation events are essential in effectuating signaling events to platelet function, we used a publicly available phosphoproteomics dataset on platelet CAMP/PKA-dependent signaling upon stimulation with ADP, iloprost or both $[15,16]$ as a starting point. We subtracted a set of proteins from the database of which we presume to be putative switch proteins or part of switch 'processes'. Hereto we made the following assumptions: 1) to specifically investigate the resolution of platelet activation, platelets must be activated first, 2) proteins involved in platelet deactivation lie downstream of the prostacyclin receptor. We started by selecting proteins that showed up- and/or downregulation upon platelet treatment with the agonist ADP for 30 seconds followed by treatment with iloprost for 30 seconds. This yielded 349 phosphosites corresponding to 215 unique proteins to be significantly regulated, see Fig.2. This first selection of phosphopeptides still contains a significant number of proteins that are involved in platelet activation through $P 2 Y_{1}$ and not $P 2 Y_{12}$ and do not necessarily play a role in platelet inhibition by iloprost (Fig.2). We therefore narrowed down our selection by omitting phosphopeptides that were regulated only after ADP stimulation. 3) We then assumed that the group of resting platelets receiving iloprost treatment had phosphorylation that can prevent or "switch off" proteins or processes involved in platelet activation. Thus, we only included peptides that were regulated when treated with iloprost, ultimately obtaining a comprehensive list of proteins with potential switches that can be "turned on" after ADP stimulation and "off" after iloprost mediated platelet inhibition. The final protein selection consisted of 30 proteins (Supplemental Table1). Twenty of these proteins had significant upregulated phosphosites while 13 proteins had significant downregulated phosphosites. The validity of this approach was supported by the identification of PDE3A, an established effector of CAMP/PKA signaling, among the 30 selected proteins. Interestingly, CNST, FGA and KALRN had both up and downregulated phosphosites. In sum, using a publicly available dataset we used knowledge about the signaling of iloprost and ADP to extract 30 proteins with potential involvement in activation and subsequent inactivation of platelets downstream CAMP/PKA.

\section{Enrichment of vesicle-related biological processes}

GO enrichment analysis can reveal overrepresented, also termed 'enriched', biological processes found in a dataset. If processes are enriched in the dataset, it means that genes annotated to a specific GO process are overrepresented compared to the entire background set. On the 30 selected proteins, GO enrichment analysis for biological processes revealed only vesicle-mediated transport to be significantly enriched. When including the additional 20 interactors from the STRING database not only vesicle-related biological processes ( 46.9 to $>100$-fold enrichment) but also positive regulation of cyclin-dependent protein kinase activity (36.3-fold enrichment), processes involving small GTPases (7.53-fold enrichment) and tight junctions (26.6-fold enrichment) were found to be enriched, see Table 1. 
Table 1

Table of significantly enriched GO biological processes. Fold enrichment with raw P-value and false discovery rate (FDR) is shown. For statistical analysis, the Fisher Exact test was used.

\begin{tabular}{|c|c|c|c|}
\hline GO biological processes & $\begin{array}{l}\text { Fold } \\
\text { enrichment }\end{array}$ & $\begin{array}{l}\text { Raw P- } \\
\text { value }\end{array}$ & FDR \\
\hline SNARE complex assembly (G0:0035493) & $>100$ & 4.77E-06 & $\begin{array}{l}6.31 \mathrm{E}- \\
03\end{array}$ \\
\hline Vesicle fusion (GO:0006906) & 46.9 & $6.63 \mathrm{E}-13$ & $\begin{array}{l}1.05 \mathrm{E}- \\
08\end{array}$ \\
\hline $\begin{array}{l}\text { Positive regulation of cyclin-dependent protein kinase activity } \\
\text { (GO:1904031) }\end{array}$ & 36.3 & 9.80E-05 & $\begin{array}{l}5.02 \mathrm{E}- \\
02\end{array}$ \\
\hline Tight junction organization (GO:0120193) & 26.6 & $1.96 \mathrm{E}-05$ & $\begin{array}{l}1.73 \mathrm{E}- \\
02\end{array}$ \\
\hline Vesicle targeting (GO:0006903) & 18.1 & 8.24E-05 & $\begin{array}{l}4.84 \mathrm{E}- \\
02\end{array}$ \\
\hline Cytosolic transport (G0:0016482) & 16.7 & $1.89 \mathrm{E}-06$ & $\begin{array}{l}3.00 \mathrm{E}- \\
03\end{array}$ \\
\hline Endosomal transport (G0:0016197) & 10.6 & $2.40 \mathrm{E}-05$ & $\begin{array}{l}1.81 \mathrm{E}- \\
02\end{array}$ \\
\hline Vesicle-mediated transport (G0:0016192) & 4.4 & 1.70E-09 & $\begin{array}{l}5.40 \mathrm{E}- \\
06\end{array}$ \\
\hline Regulation of protein localization (G0:0032880) & 4.31 & 8.95E-05 & $\begin{array}{l}4.89 \mathrm{E}- \\
02\end{array}$ \\
\hline $\begin{array}{l}\text { Regulation of small GTPase mediated signal transduction } \\
\text { (GO:0051056) }\end{array}$ & 7.53 & 1.50E-04 & $\begin{array}{l}6.24 \mathrm{E}- \\
02\end{array}$ \\
\hline
\end{tabular}

Network analysis

Phosphopeptide regulation

The protein-protein interaction network was created with the 30 selected proteins mentioned under 'Data selection and characterization'. We added 20 additional interactors to include proteins that are likely to play a role in the major biological processes covered by the network. Ultimately, it contained 30 regulated proteins, 20 additional interactors and 79 edges connecting the nodes (Fig. 3). Regulated phosphosites according to Beck et al. $[15,16]$ - were visualized on the nodes with pie charts and node size was mapped to indicate the total number of phosphosites found in each protein. The network contained 4 major clusters consisting of 4 or more nodes. 13 out of 15 nodes from cluster A (Fig. 3a) are related to vesiclemediated transport and 6 out of 9 nodes from the cluster B (Fig. 3b) are related to cell shape or regulation of small GTPase mediated signal transduction. The smaller clusters are related to cyclin-dependent kinases (Fig. 3c) and tight junctions (Fig. 3d) and contain less than 6 nodes. Fourteen proteins did not 
have protein interactions within the constructed network (Fig. 3e). The topological clusters in the network correspond with the results from the enrichment analysis (Table 1), indicating that these are functional clusters related to specific biological processes.

PKA phosphorylation scores and quantitative platelet proteome

We hypothesized that phosphorylation by PKA plays a role in regulating molecular switches of platelet activity and inhibition. Proteins with a high likelihood of being phosphorylated by PKA were identified with the GPS algorithm and visualized as blue nodes in an alternative representation of the network (Fig. 4). As expected, almost all of the 30 proteins in our original list had GPS scores above the highest threshold, as indicated by the GPS tool, and matched with the found phosphorylation sites of the dataset except for MARCH2. Interestingly, several interactors added using StringApp, such as RAC1, VTI1B and CCNY, also had GPS scores above this threshold. The estimated copy number per platelet, based on mass spectrometric (MS) quantitation by Burkhart et al. [26], was incorporated in the network visualization as node size (Fig. 4). Twelve proteins did not have an estimated copy number, which is indicated with a red outline in Fig. 4. Of these 12 proteins, 1-phosphatidylinositol 3-phosphate 5-kinase (PIKFYVE) and transGolgi network integral membrane protein 2 (TGOLN2) were present in the initial dataset, suggesting that these proteins are present in platelets but below the MS detection limit. The 10 other proteins without an estimated copy number are either absent in platelets or present below the MS detection limit. No quantitative data was available for VAMP4 (Fig. 4a) and RHOB (Fig. 4b) as they were only detectable after enrichment. In the cluster corresponding to tight junctions, no proteins other than CLDN5 were detected in the platelet proteome (Fig. 4c).

Relative expression of corresponding genes

To obtain an indication of the relative expression of proteins in our network in hematopoietic cells, we used the publicly available database Blood RNAexpress [27] (Fig. 5). For reference, the gene GP1BA, coding for part of the GP1b receptor and well known to be abundant in platelets, had an average relative normalized gene expression, log2 fpkm, of over 9 . All genes had a log2 fpkm higher than 1, except for FGA, OR14A2, CLDN4/22/25 and REC114 (named C15orf60 in dataset). Several genes revealed by our analysis; SLAIN2, MARCH2, VAMP3, ARPP19, STON2, PHKB, ABLIM3, and IMUP (named C19orf33 in dataset) had a log2 fpkm higher than 9 (Supplemental Table 2). This indicates that these genes are more abundantly expressed in platelets when compared to other hematopoietic cells.

Integration of DrugBank database

Drug-target interactions were integrated into the network to give a clear and quick overview of current FDA-approved drugs that have one of the proteins in the network as target. In Fig. 6, blue diamonds represent drugs and orange shapes display their targets. In the largest cluster related to vesicle-mediated transport, FGA stood out as a protein targeted by 18 drugs. In the smaller cluster representing mostly proteins involved in cell shape or regulation of small GTPase mediated signal transduction, RHOB and RAC1 were targeted by botulinum toxin type A and azathioprine, respectively. PDE3A, although not 
connected to the rest of the network, was in the initial selection of potential "switch" proteins and was targeted by 11 drugs.

\section{Discussion}

In this study we developed a network biology approach to find putative novel switches in the cAMP-PKA signaling pathway in platelets. The network analyses were automated in $\mathrm{R}$ and can be adapted to include different proteins or an entirely different dataset. Our method can be used to repurpose existing datasets and provide a coherent overview of mechanisms involved to predict novel connections, by visually integrating multiple datasets. Here, this method was utilized to distil proteins from an existing phosphoproteomics dataset containing both activation and inhibition of platelets $[15,16]$. Proteins not related to phosphorylation were inherently not included in the original phosphoproteomics datasets, as well as proteins excluded by our stringent selection, e.g., effectors downstream PKA after only ADP stimulation. However, such candidates might still be included through the addition of interactors using tools like the StringApp. Furthermore, although cAMP-PKA signaling is a major platelet inhibitory pathway, other pathways such as NO-cGMP/PKG signaling inhibit platelet activation. An advantage of our workflow is that it is designed in such a way that it could be readily adapted to process an alternate data selection or an entirely different dataset. Following our rationale, we were able to extract a subset of 30 proteins from this dataset, i.e., those proteins displaying both altered phosphorylation status upon sequential treatment of platelets with ADP plus iloprost and altered phosphorylation by iloprost treatment only. We postulate that the activity of these 30 proteins can be modified after ADP-mediated platelet activation and subsequently remodified after platelet inhibition with iloprost.

GO enrichment analysis revealed that multiple biological processes related to vesicle secretion, regulation of small GTPases and regulation of cyclin-dependent protein kinase activity were significantly overrepresented in our obtained list of 30 proteins. These processes are known to be important in platelet physiology. Platelets influence hemostasis by secreting granules and communicate with their environment by producing extracellular vesicles. Small GTPases play a pivotal in the control of vesicle trafficking and platelet aggregation or thrombus formation via $a_{\| b} \beta_{3}$. Network analysis showed several clusters corresponding to these enriched biological processes to be well defined. We will discuss regulated proteins that are detected/detectable in the platelet proteome (27 out of 30, Fig. 4) and have a high GPS score for PKA according to the cut-off of the GPS tool, as our hypothesis is that downstream of the cAMP-PKA pathway proteins or processes are present that can serve as a "switch" in platelet activation and inhibition.

The largest cluster in our analysis is related to vesicle-mediated transport. Interesting proteins in this cluster included FGA, STXBP5, STON2, SEC22B, LRMP, and VTI1B. The role of these 6 proteins in platelet function has well been described for fibrinogen alpha chain (FGA) and less well for the others, as fibrin is a major constituent of thrombi and essential to normal hemostasis [29]. Syntaxin-binding protein 5 (STXBP5) has contradicting functions in platelets and endothelial cells, it promotes granule secretion in platelets but inhibits exocytosis in endothelial cells [30]. A single-nucleotide polymorphism in the STXBP5 
locus has also been associated with a decreased thrombotic phenotype [31]. In this study STXBP5 is indicated as a putative PKA substrate, which is in line with other phosphoproteomics studies on resting platelets [32]. Stonin-2 (STON2) is involved in the endocytic machinery, synaptic vesicle recycling and clathrin coated vesicle uncoating [33]. To our knowledge Stonin-2's function has not been described in platelets even though Stonin-2 mRNA expression is relatively high in platelets compared to other hematopoietic cells, making it an interesting target for future research. SEC22B is a membrane-resident trafficking protein that is required for $\otimes$-granule production in megakaryocytes and can interact with NBEAL2, which is associated with grey platelet syndrome. LRMP better known as inositol 1,4,5triphosphate receptor associated 2 (IRAG2), interacts with the inositol 1,4,5-triphosposphate receptor in mice indicating a potential role in calcium homeostasis [34]. Platelet endocytosis/exocytosis is important in e.g., loading/release of a-granules. Studies have also implicated that integrin trafficking contributes to platelet activation and thrombosis by controlling their surface expression [35]. As more mechanistic studies show platelet endocytosis to be involved in platelet function [36], it is interesting to see a pronounced representation in our network analysis.

The second largest cluster of proteins was related to regulation of small GTPase mediated signal transduction and cell shape. This cluster included ARHGEF6, PPP1R14A, KALRN, MYO9B, and ABLIM3, all with a high PKA score. Not much is known about ABLIM3's function in platelets. However, the other proteins have been studied extensively in relation to platelet function. ARHGEF6 has previously been reported to be a substrate for PKA and PKG and acts as a mediator in reducing Rac1-GTP levels leading to less outside-in platelet signaling [37]. Protein phosphatase 1 regulatory subunit 14A (PPP1R14A or CPI17 ) is a phosphorylation-dependent inhibitor protein of myosin phosphatase. Phosphorylation at Thr-38 causes a conformational change that greatly increases its inhibitory potential [38]. Although phosphorylation happens mainly through PKC and Rho-associated protein kinase (ROCK), it has a potential PKA target consensus sequence, as indicated by the GPS 5.0 algorithm. Phosphorylation of PPP1R14A has been shown to regulate shape change in platelets trough calcium-independent signaling pathways. Platelet shape change is necessary for complete platelet activation. PPP1R14A could therefore be involved in a potential switch in platelet hyperreactivity as it can regulate myosin light chain phosphatase [39]. Another protein related to Rac/Rho protein signaling is kalirin (KALRN). In platelets, adenosine 5'-diphosphate-ribosylation factor 6 (ARF6) controls platelet spreading via integrin $\mathrm{a}_{11 \mathrm{~b}} \beta_{3}$ trafficking and can recruit KALRN to the plasma membrane leading to Rac activation $[35,40]$. The ATP binding site within KALRN might be used as a starting point in the design of specific inhibitors. Inhibition of Rac1 by small molecules has been studied in the context of platelet secretion as a potential future antiplatelet drug [41]. MYO9B has recently been indicated to regulate RhoA activation though phosphorylation by PKA and PKG [42]. RhoA is a molecular switch controlled by GTPases. Platelet activation by ADP in RhoA knockout mice results in defective platelet function and unstable thrombus formation [43]. RAC1 and RHOB were central nodes interacting with most of these proteins. Rho GTPases are known key regulators of platelet cytoskeleton and platelet function and serve as molecular switches downstream of platelet surface receptors. It is therefore expected that these proteins emerge as potential candidate switches for platelet regulation from our selection. 
The cluster related to cyclin-dependent kinases contained at least three interesting proteins all with significantly upregulated phosphorylation sites after ADP and iloprost treatment; CDK16/17 and Cyclin-Y (CCNY). Regulation of cyclin-dependent protein kinase activity might seem surprising at first sight as cyclin-dependent proteins belong to a class of proteins involved in cell cycle, transcription and mRNA processing and platelets do not have a nucleus and limited mRNA processing capacity [44]. However, recent focus on the role of non-coding RNA, nuclear receptors and post-transcriptional modifications in platelets reveal a more intricate transcriptional landscape than previously thought [45, 46]. In HEK293a cells, CDK16 is recruited to the plasma membrane and activated by CNNY. This process is regulated by Ser-153 and can be inhibited by PKA phosphorylation [47]. In line with these findings, we found Ser-153 to be a putative PKA phosphorylation site. Platelets lacking CCNY show decreased spreading and clot retraction, but increased adhesion to collagen [48]. Taken together, these new findings suggest that CCNY could play an important role in the outside-in signaling in platelets.

Finally, the smaller clusters and single nodes in our network also contain interesting proteins that are worth mentioning. PDE3A, CLDN5, GCSAML, MTSS1, ARPP19, MACF1, TBC1D23, UBE20, GAS2L1, PHKB, SCAMP3, CNST, RGCC, SLAIN2, and EIF3B. As expected, among the 30 proteins revealed by pathway analysis, we found proteins known to be involved in the cAMP-PKA signaling axis, e.g., PDE3A. While iloprost causes cAMP levels to go up, PDE3A hydrolyses cAMP. In diabetes mellitus, platelet hyperreactivity is believed to at least partly be caused by reduced platelet sensitivity to insulin, which leads to decreased endothelial $\mathrm{PGI}_{2}$ expression, increased $\mathrm{P} 2 \mathrm{Y}_{12}$-mediated $\mathrm{Gi}$ activity and decreased platelet cAMP levels, thus leading to increased platelet activation $[49,50]$. PKA-induced phosphorylation of PDE3A creates a negative feedback loop, indicating PDE3A being a key element in the cAMP/PKA pathway at least in initiation of platelet activation [51]. Although not directly connected to any other nodes in the protein network, it is foreseeable that PDE3A is returned from the analysis as an interesting target. In fact, the validity of the method presented in this study can be inferred by its identification of PDE3A.

As more large datasets are generated daily by innovative studies utilizing high-throughput methods, integration of these datasets to put them in a specific context are necessary. Finding novel players in platelet activation/inhibition can help us better understand pathologies where platelet hyperreactivity is prevalent. Further experimental studies using a more causal analysis approach can reveal what effect a phosphorylation event has on a protein's function [52]. Experimental knockout mice or using inhibitors in flow experiments for the aforementioned proteins are valid options to further validate the roles of these proteins in platelet hyperreactivity. Finally, this study shows the importance and benefit of data integration and visualization with existing tools and datasets to obtain a complete picture of complex molecular mechanisms involved.

\section{Declarations}

\section{Ethics approval and consent to participate}




\section{Consent to publish}

Not applicable

\section{Availability of data and materials}

The phosphoproteomics datasets analysed during the current study are available through

ProteomeXchange under the accession PXD000242 and PXD001189. Data on the platelet proteome can be found in supplementary information from [Burkhart et. al., Blood 2012]. Relative RNA expression data is available from https://blueprint.haem.cam.ac.uk/mRNA/.

\section{Competing interests}

The authors have no conflicts of (financial) interest to declare. All co-authors have seen and agree with the contents of the article.

\section{Funding}

This work was supported by the Netherlands Foundation for Scientific Research (ZonMW VIDI 91716421 to T.P.L. and J.M.E.M.C.), the Dutch Heart Foundation (2015T79 to DMC and J.M.E.M.C.) and the Landsteiner Foundation for Blood Transfusion Research (LSBR Nr. 1638 to R.R.K.).

\section{Authors' Contributions}

All authors read and approved the final manuscript. TL and MK have performed the analysis. TL has written the manuscript text. All other authors have provided a significant contribution to the design of the workflow or interpretations of the obtained results.

\section{Acknowledgements}

Not applicable

\section{Abbreviations}

ABLIM Actin-binding LIM protein

AC adenylate cyclase 


\begin{tabular}{|c|c|}
\hline ADP & adenosine diphosphate \\
\hline ARF6 & adenosine 5'-diphosphate-ribosylation factor 6 \\
\hline ARPP19 & cAMP-regulated phosphoprotein 19 \\
\hline CABLES1 & CDK5 and ABL1 enzyme substrate 1 \\
\hline CalDAG-GEFI & calcium- and DAG-regulated guanine exchange factor- 1 \\
\hline CAMP & cyclic adenosine monophosphate \\
\hline CCNY & Cyclin-Y \\
\hline CLDN & Claudin \\
\hline CNST & Consortin \\
\hline FGA & Fibrinogen alpha chain \\
\hline GO & Gene Ontology \\
\hline GPCR & G protein-coupled receptors \\
\hline IMUP & Immortalization up-regulated protein \\
\hline KALRN & Kalirin \\
\hline MARCH2 & E3 ubiquitin-protein ligase MARCHF2 \\
\hline NO & nitric oxide \\
\hline OR14A2 & Olfactory receptor $14 \mathrm{~A} 2$ \\
\hline PDE3A & Phosphodiesterase $3 \mathrm{~A}$ \\
\hline PGI2 & prostaglandin 12 \\
\hline PHKB & Phosphorylase b kinase regulatory subunit beta \\
\hline $\mathrm{PI} 3 \mathrm{~K}$ & phosphoinositide 3-kinase \\
\hline PIKFYVE & 1-phosphatidylinositol 3-phosphate 5-kinase \\
\hline PKA & protein kinase A \\
\hline PPP1R14A & Protein phosphatase 1 regulatory subunit $14 \mathrm{~A}$ \\
\hline
\end{tabular}


RAC1 $\quad$ Ras-related C3 botulinum toxin substrate 1

RASA3 Ras GTPase-activating protein 3

REC114 Meiotic recombination protein REC114

RHOB Rho-related GTP-binding protein RhoB

ROCK Rho-associated protein kinase

SLAIN2 SLAIN motif-containing protein 2

STON2 Stonin-2

T2DM type 2 diabetes mellitus

TGOLN2 Trans-Golgi network integral membrane protein 2

VAMP Vesicle-associated membrane protein

VTI1B Vesicle transport through interaction with t-SNAREs homolog 1B

\section{References}

1. Konukoglu D, Uzun H: Endothelial Dysfunction and Hypertension. In: Hypertension: from basic research to clinical practice. Springer International Publishing; 2017: 511-540.

2. Davignon J, Ganz P: Role of Endothelial Dysfunction in Atherosclerosis. Circulation 2004, 109:2732.

3. Tabit CE, Chung WB, Hamburg NM, Vita JA: Endothelial dysfunction in diabetes mellitus: molecular mechanisms and clinical implications. Rev Endocr Metab Disord 2010, 11(1):61-74.

4. Coenen DM, Heinzmann ACA, Karel MFA, Cosemans JMEM, Koenen RR: The multifaceted contribution of platelets in the emergence and aftermath of acute cardiovascular events. Atherosclerosis.

5. Nissen SE, Nicholls SJ, Wolski K, Nesto R, Kupfer S, Perez A, Jure H, De Larochellière R, Staniloae CS, Mavromatis K et al: Comparison of Pioglitazone vs Glimepiride on Progression of Coronary Atherosclerosis in Patients With Type 2 Diabetes: The PERISCOPE Randomized Controlled Trial. JAMA 2008, 299(13):1561-1573.

6. Kahal H, Aburima A, Spurgeon B, Wraith KS, Rigby AS, Sathyapalan T, Kilpatrick ES, Naseem KM, Atkin SL: Platelet function following induced hypoglycaemia in type 2 diabetes. Diabetes \& Metabolism 2018, 44(5):431-436.

7. Matetzky S, Shenkman B, Guetta V, Shechter M, Beinart R, Goldenberg I, Novikov I, Pres H, Savion N, Varon $\mathrm{D}$ et al: Clopidogrel resistance is associated with increased risk of recurrent atherothrombotic 
events in patients with acute myocardial infarction. Circulation 2004, 109(25):3171-3175.

8. Michelson AD, Linden MD, Furman MI, Li Y, Barnard MR, Fox ML, Lau WC, McLaughlin TJ, Frelinger $A L$ : Evidence that pre-existent variability in platelet response to ADP accounts for 'clopidogrel resistance'. J Thromb Haemost 2007, 5(1):75-81.

9. Cuisset T, Frere C, Quilici J, Barbou F, Morange PE, Hovasse T, Bonnet JL, Alessi MC: High posttreatment platelet reactivity identified low-responders to dual antiplatelet therapy at increased risk of recurrent cardiovascular events after stenting for acute coronary syndrome. J Thromb Haemost 2006, 4(3):542-549.

10. Kamea T, Shiraga M, Kahsiwagi H, Kato H, Tadokoro S, Kurata Y, Tomiyama Y, Kanakura Y: Critical role of ADP interaction with P2Y12 receptor in the maintenance of allb $\beta 3$ activation: association with Rap1B activation. Journal of Thrombosis and Haemostasis 2006, 4(6):1379-1387.

11. Léon C, Hechler B, Freund M, Eckly A, Vial C, Ohlmann P, Dierich A, LeMeur M, Cazenave JP, Gachet C: Defective platelet aggregation and increased resistance to thrombosis in purinergic $\mathrm{P} 2 \mathrm{Y}(1)$ receptornull mice. J Clin Invest 1999, 104(12):1731-1737.

12. Chrzanowska-Wodnicka M, Smyth SS, Schoenwaelder SM, Fischer TH, White GC, 2nd: Rap1b is required for normal platelet function and hemostasis in mice. J Clin Invest 2005, 115(3):680-687.

13. Cattaneo M: 14 - The Platelet P2 Receptors. In: Platelets (Fourth Edition). Academic Press; 2019 : $259-277$.

14. Stefanini L, Paul DS, Robledo RF, Chan ER, Getz TM, Campbell RA, Kechele DO, Casari C, Piatt R, Caron KM et al: RASA3 is a critical inhibitor of RAP1-dependent platelet activation. J Clin Invest 2015, 125(4):1419-1432.

15. Beck F, Geiger J, Gambaryan S, Solari FA, Dell'Aica M, Loroch S, Mattheij NJ, Mindukshev I, Pötz O, Jurk K et al: Temporal quantitative phosphoproteomics of ADP stimulation reveals novel central nodes in platelet activation and inhibition. Blood 2017, 129(2):e1-e12.

16. Beck F, Geiger J, Gambaryan S, Veit J, Vaudel M, Nollau P, Kohlbacher O, Martens L, Walter U, Sickmann A et al: Time-resolved characterization of cAMP/PKA-dependent signaling reveals that platelet inhibition is a concerted process involving multiple signaling pathways. Blood 2014, 123(5):e1-e10.

17. Doncheva NT, Morris JH, Gorodkin J, Jensen LJ: Cytoscape StringApp: Network Analysis and Visualization of Proteomics Data. J Proteome Res 2019, 18(2):623-632.

18. Smoot ME, Ono K, Ruscheinski J, Wang P-L, Ideker T: Cytoscape 2.8: new features for data integration and network visualization. Bioinformatics 2011, 27(3):431-432.

19. Gustavsen J, Pai S, Isserlin R, Demchak B, Pico A: RCy3: Network biology using Cytoscape from within R [version 3; peer review: 3 approved]. F1000Research 2019, 8(1774).

20. Ashburner M, Ball CA, Blake JA, Botstein D, Butler H, Cherry JM, Davis AP, Dolinski K, Dwight SS, Eppig JT et al: Gene ontology: tool for the unification of biology. The Gene Ontology Consortium. Nat Genet 2000, 25(1):25-29. 
21. The Gene Ontology Consortium: The Gene Ontology Resource: 20 years and still GOing strong. Nucleic Acids Res 2019, 47(D1):D330-D338.

22. $\mathrm{Mi} \mathrm{H}$, Muruganujan $\mathrm{A}$, Casagrande $\mathrm{JT}$, Thomas $\mathrm{PD}$ : Large-scale gene function analysis with the PANTHER classification system. Nat Protoc 2013, 8(8):1551-1566.

23. Xue Y, Liu Z, Cao J, Ma Q, Gao X, Wang Q, Jin C, Zhou Y, Wen L, Ren J: GPS 2.1: enhanced prediction of kinase-specific phosphorylation sites with an algorithm of motif length selection. Protein Eng Des Sel 2011, 24(3):255-260.

24. Wishart DS, Feunang YD, Guo AC, Lo EJ, Marcu A, Grant JR, Sajed T, Johnson D, Li C, Sayeeda Z et al: DrugBank 5.0: a major update to the DrugBank database for 2018. Nucleic Acids Res 2018, 46(D1):D1074-D1082.

25. Kutmon M, Kelder T, Mandaviya P, Evelo CTA, Coort SL: CyTargetLinker: a cytoscape app to integrate regulatory interactions in network analysis. PLoS One 2013, 8(12):e82160.

26. Burkhart JM, Vaudel M, Gambaryan S, Radau S, Walter U, Martens L, Geiger J, Sickmann A, Zahedi RP: The first comprehensive and quantitative analysis of human platelet protein composition allows the comparative analysis of structural and functional pathways. Blood 2012, 120(15):e73-82.

27. Chen L, Kostadima M, Martens JHA, Canu G, Garcia SP, Turro E, Downes K, Macaulay IC, BielczykMaczynska E, Coe S et al: Transcriptional diversity during lineage commitment of human blood progenitors. Science 2014, 345(6204):1251033.

28. Li Z, Smyth SS: 16 - Interactions Between Platelets, Leukocytes, and the Endothelium. In: Platelets (Fourth Edition). Academic Press; 2019: 295-310.

29. Swieringa F, Baaten CCFMJ, Verdoold R, Mastenbroek TG, Rijnveld N, Laan KOvd, Breel EJ, Collins PW, Lancé MD, Henskens YMC et al: Platelet Control of Fibrin Distribution and Microelasticity in Thrombus Formation Under Flow. Arteriosclerosis, Thrombosis, and Vascular Biology 2016, 36(4):692-699.

30. Zhu Q, Yamakuchi M, Ture S, la Luz Garcia-Hernandez Md, Ko KA, Modjeski KL, LoMonaco MB, Johnson AD, O'Donnell CJ, Takai Y et al: Syntaxin-binding protein STXBP5 inhibits endothelial exocytosis and promotes platelet secretion. J Clin Invest 2014, 124(10):4503-4516.

31. Zhu QM, Ko KA, Ture S, Mastrangelo MA, Chen MH, Johnson AD, O'Donnell CJ, Morrell CN, Miano JM, Lowenstein CJ: Novel Thrombotic Function of a Human SNP in STXBP5 Revealed by CRISPR/Cas9 Gene Editing in Mice. Arterioscler Thromb Vasc Bio/ 2017, 37(2):264-270.

32. Zahedi RP, Lewandrowski U, Wiesner J, Wortelkamp S, Moebius J, Schütz C, Walter U, Gambaryan S, Sickmann A: Phosphoproteome of Resting Human Platelets. Journal of Proteome Research 2008, 7(2):526-534.

33. Martina JA, Bonangelino CJ, Aguilar RC, Bonifacino JS: Stonin 2: an adaptor-like protein that interacts with components of the endocytic machinery. J Cell Biol 2001, 153(5):1111-1120.

34. Shindo Y, Kim M-R, Miura H, Yuuki T, Kanda T, Hino A, Kusakabe Y: Lrmp/Jaw1 is expressed in sweet, bitter, and umami receptor-expressing cells. Chem Senses 2010, 35(2):171-177. 
35. Huang Y, Joshi S, Xiang B, Kanaho Y, Li Z, Bouchard BA, Moncman CL, Whiteheart SW: Arf6 controls platelet spreading and clot retraction via integrin allbß3 trafficking. Blood 2016, 127(11):1459-1467.

36. Banerjee $M$, Whiteheart SW: The ins and outs of endocytic trafficking in platelet functions. Curr Opin Hematol 2017, 24(5):467-474.

37. Nagy Z, Wynne K, von Kriegsheim A, others: Cyclic nucleotide-dependent protein kinases target ARHGAP17 and ARHGEF6 complexes in platelets. Journal of Biological 2015.

38. Eto M, Kitazawa T, Matsuzawa F, Aikawa S-I, Kirkbride JA, Isozumi N, Nishimura Y, Brautigan DL, Ohki S-Y: Phosphorylation-induced conformational switching of CPI-17 produces a potent myosin phosphatase inhibitor. Structure 2007, 15(12):1591-1602.

39. Aslam M, Härtel FV, Arshad M, Gündüz D, Abdallah Y, Sauer H, Piper HM, Noll T: cAMP/PKA antagonizes thrombin-induced inactivation of endothelial myosin light chain phosphatase: role of CPI-17. Cardiovasc Res 2010, 87(2):375-384.

40. Koo TH, Eipper BA, Donaldson JG: Arf6 recruits the Rac GEF Kalirin to the plasma membrane facilitating Rac activation. BMC Cell Bio/ 2007, 8:29.

41. Dwivedi S, Pandey D, Khandoga AL, Brandl R, Siess W: Rac1-mediated signaling plays a central role in secretion-dependent platelet aggregation in human blood stimulated by atherosclerotic plaque. $J$ Transl Med 2010, 8:128.

42. Comer S, Nagy Z, Bolado A, von Kriegsheim A, Gambaryan S, Walter U, Pagel O, Zahedi RP, Jurk K, Smolenski A: The RhoA regulators Myo9b and GEF-H1 are targets of cyclic nucleotide-dependent kinases in platelets. Journal of Thrombosis and Haemostasis 2020, 18(11):3002-3012.

43. Pleines I, Hagedorn I, Gupta S, May F, Chakarova L, van Hengel J, Offermanns S, Krohne G, Kleinschnitz C, Brakebusch $C$ et al: Megakaryocyte-specific RhoA deficiency causes macrothrombocytopenia and defective platelet activation in hemostasis and thrombosis. Blood 2012, 119(4):1054-1063.

44. Ventura E, Giordano A: Cell Cycle. In: Reference Module in Life Sciences. Elsevier; 2019.

45. Bray PF, McKenzie SE, Edelstein LC, Nagalla S, Delgrosso K, Ertel A, Kupper J, Jing Y, Londin E, Loher $\mathrm{P}$ et al: The complex transcriptional landscape of the anucleate human platelet. BMC Genomics 2013, 14(1):1.

46. Schwertz H, Rowley JW, Zimmerman GA, Weyrich AS, Rondina MT: Retinoic acid receptor-a regulates synthetic events in human platelets. J Thromb Haemost 2017, 15(12):2408-2418.

47. Mikolcevic P, Sigl R, Rauch V, Hess MW, Pfaller K, Barisic M, Pelliniemi LJ, BoesI M, Geley S: Cyclindependent kinase 16/PCTAIRE kinase 1 is activated by cyclin $Y$ and is essential for spermatogenesis. Mol Cell Biol 2012, 32(4):868-879.

48. Kyselova A, Siragusa M, Anthes J, Solari FA, Loroch S, Zahedi RP, Walter U, Fleming I, Randriamboavonjy V: Cyclin $\mathrm{Y}$ is expressed in Platelets and Modulates Integrin Outside-in Signaling. Int J Mol Sci 2020, 21(21).

49. Morel O, Jesel L, Abbas M, Morel N: Prothrombotic changes in diabetes mellitus. Semin Thromb Hemost 2013, 39(5):477-488. 
50. Kaur R, Kaur M, Singh J: Endothelial dysfunction and platelet hyperactivity in type 2 diabetes mellitus: molecular insights and therapeutic strategies. Cardiovasc Diabetol 2018, 17(1):121.

51. Smolenski A: Novel roles of cAMP/cGMP-dependent signaling in platelets: CAMP/cGMP-signaling in platelets. J Thromb Haemost 2012, 10(2):167-176.

52. Babur Ö, Melrose AR, Cunliffe JM, Klimek J, Pang J, Sepp A-LI, Zilberman-Rudenko J, Tassi Yunga S, Zheng T, Parra-Izquierdo I et al: Phosphoproteomic quantitation and causal analysis reveal pathways in GPVI/ITAM-mediated platelet activation programs. Blood 2020, 136(20):2346-2358.

\section{Figures}

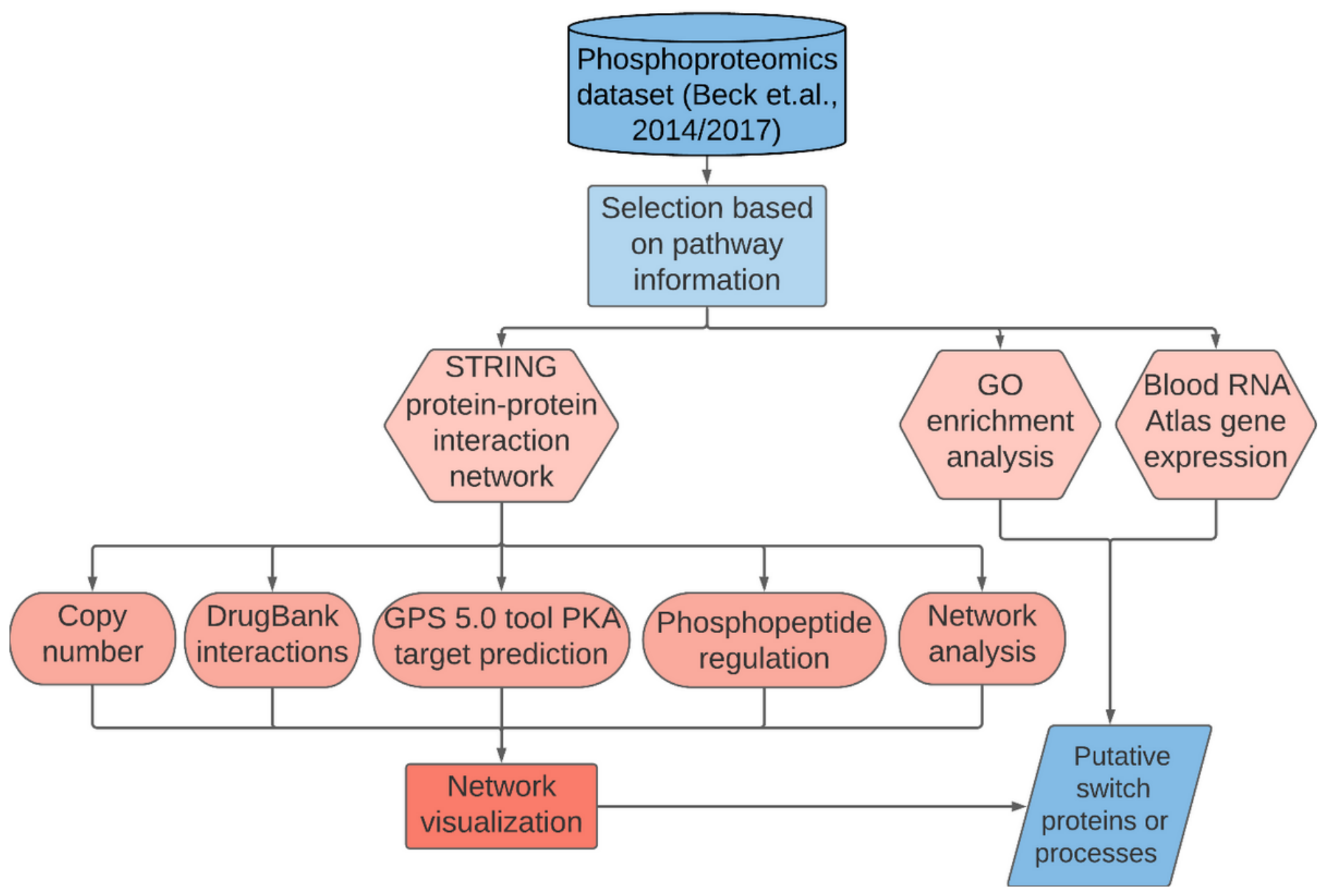

Figure 1

Schematic overview of the methodological workflow. This workflow was used to find switches in platelet activation and inhibition downstream of cAMP/PKA signaling using a publicly available phosphoproteomics dataset $[15,16]$. 


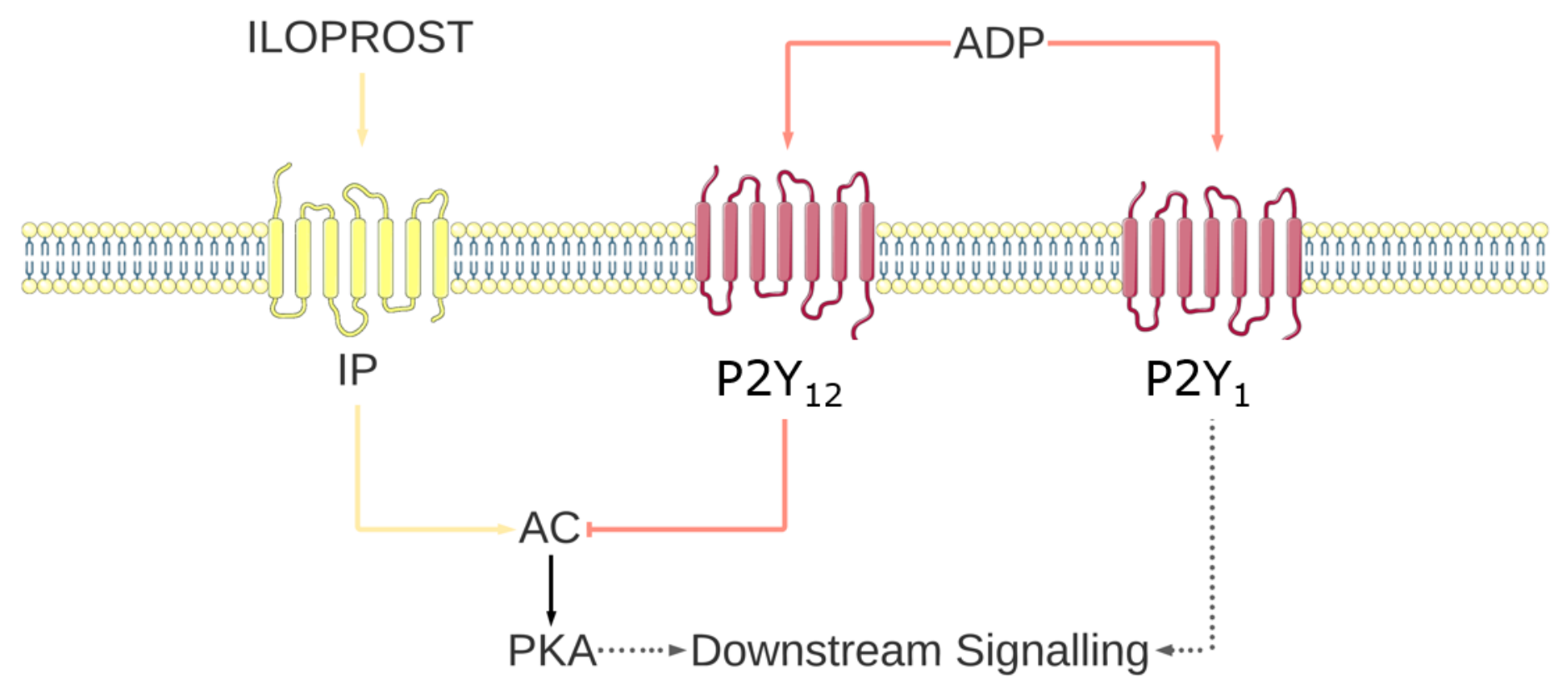

\section{Phosphoproteomics Datasets}

Regulated proteins after 30s ADP + 30s Iloprost

To include proteins that are involved in ADP-dependent platelet activation and deactivation downstream PKA

\section{Omitting proteins only regulated after 30 s ADP}

To include proteins involved in the resolution of platelet activation

Only including proteins that are also regulated after just 30s Iloprost

To include proteins that putatively can be switched on AND off

\section{Figure 2}

Summary of pathway information and protein selection. After 30 seconds ADP stimulation and subsequent 30 seconds iloprost treatment, 349 phosphosites corresponding to 215 unique proteins were significantly regulated. Omitting those regulated only after just 30 seconds ADP stimulation resulted in 73 proteins. Cross-referencing these 73 proteins with those regulated after just 30 seconds iloprost treatment resulted in a final selection of 30 proteins. 

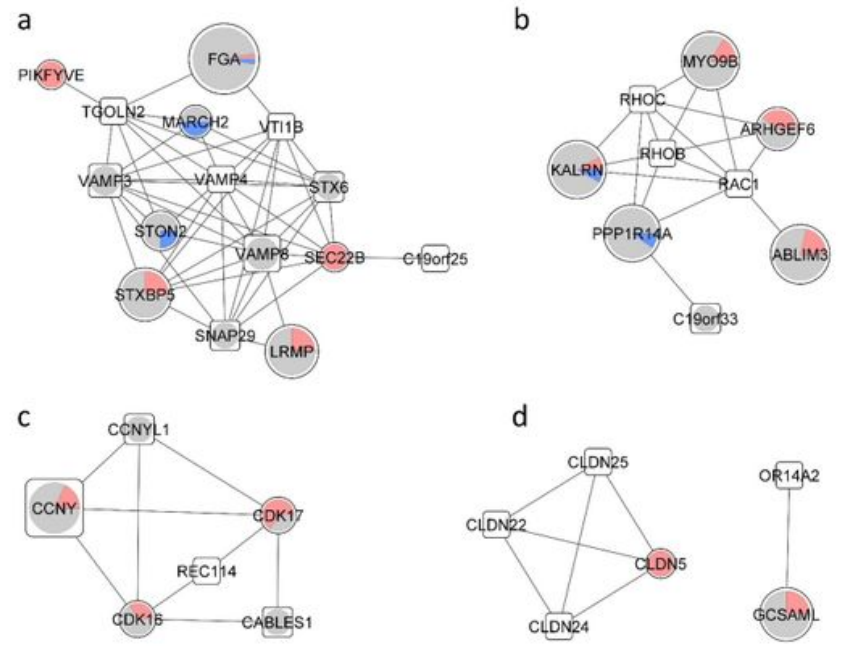

d

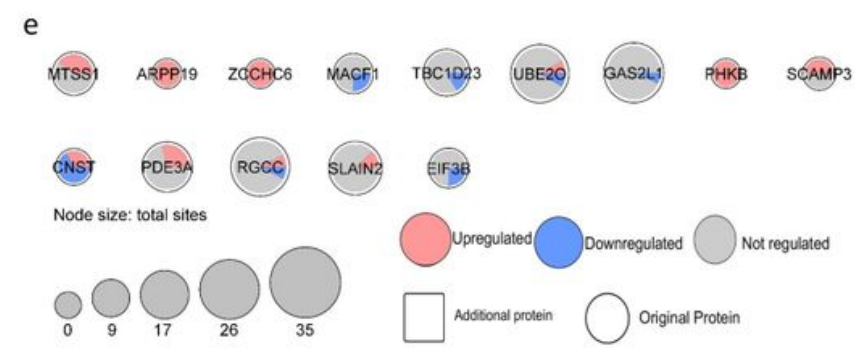

\section{Figure 3}

Protein-protein interaction network of potential "switch" candidates in platelet activation. Total number of phosphorylation sites as reported by Beck et. al. [16] are depicted as representative node sizes and regulation is visualized by either red or blue for upregulated or downregulated sites, respectively. Proteins added to the network by StringApp are represented by squares. Corresponding gene names are indicated per node. Cluster of proteins related to (a) vesicle-mediated transport, (b) regulation of small GTPase mediated signal transduction and cell shape, (c) cyclin-dependent kinases, and (d) tight junctions. (e) Single nodes representing proteins that do not have direct interactions with other proteins in the network.

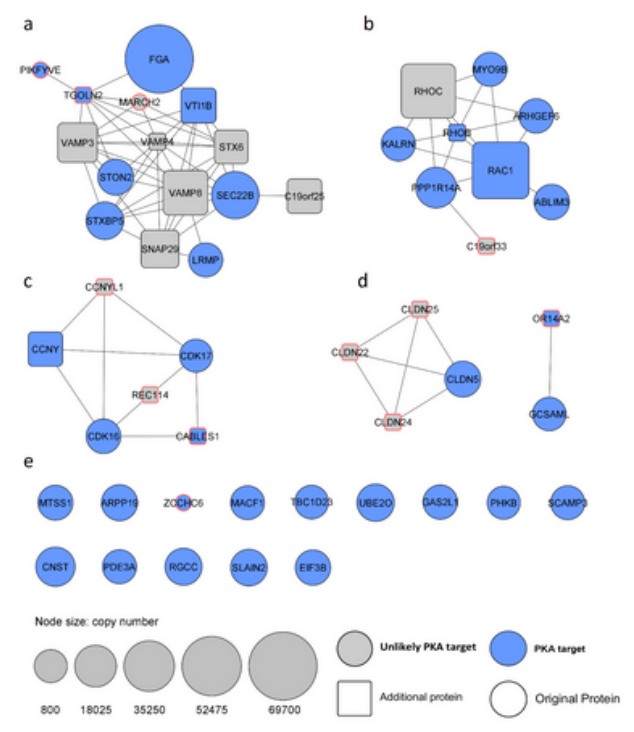




\section{Figure 4}

Protein-protein interaction network of potential "switch" candidates in platelet activation. Estimated copy numbers are visualized as node size and the fill color indicates potential PKA substrates. Red outline indicates no known copy number in platelets. Proteins added to the network by StringApp are represented by squares. Cluster of proteins related to (a) vesicle-mediated transport, (b) regulation of small GTPasesmediated signal transduction and cell shape, (c) cyclin-dependent kinases, and (d) tight junctions. (e) Single and double nodes represent proteins that do not have direct interactions with other proteins in the network.

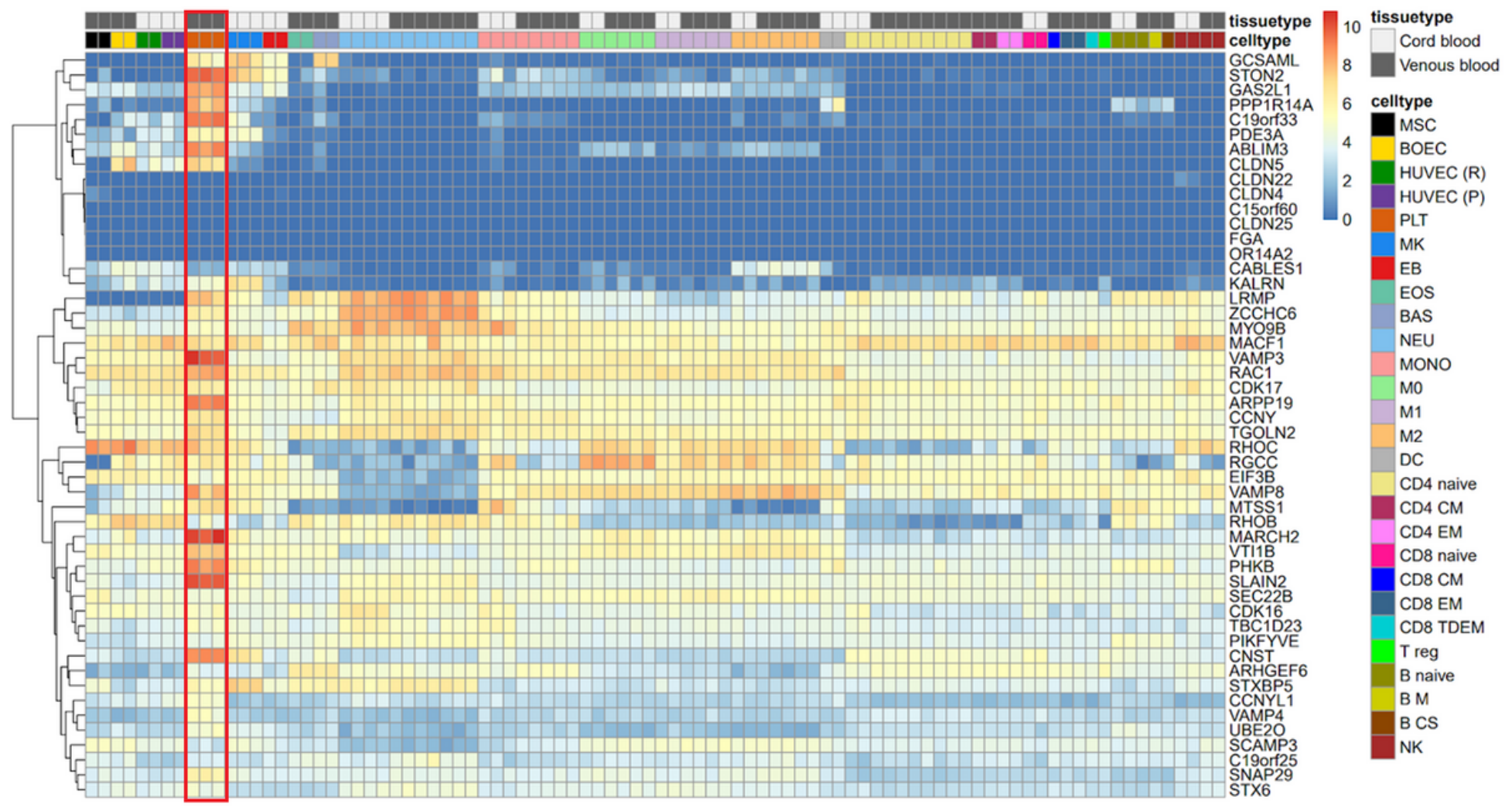

Figure 5

Clustered heatmap of relative hematopoietic cell-specific RNA expression of genes in the network. Orange color marks platelet cell type RNA expression relative to other hematopoietic cell populations (log2 fpkm). Red indicates high relative expression and blue indicates low relative expression. Data source and further information on: https://blueprint.haem.cam.ac.uk/mRNA/ 


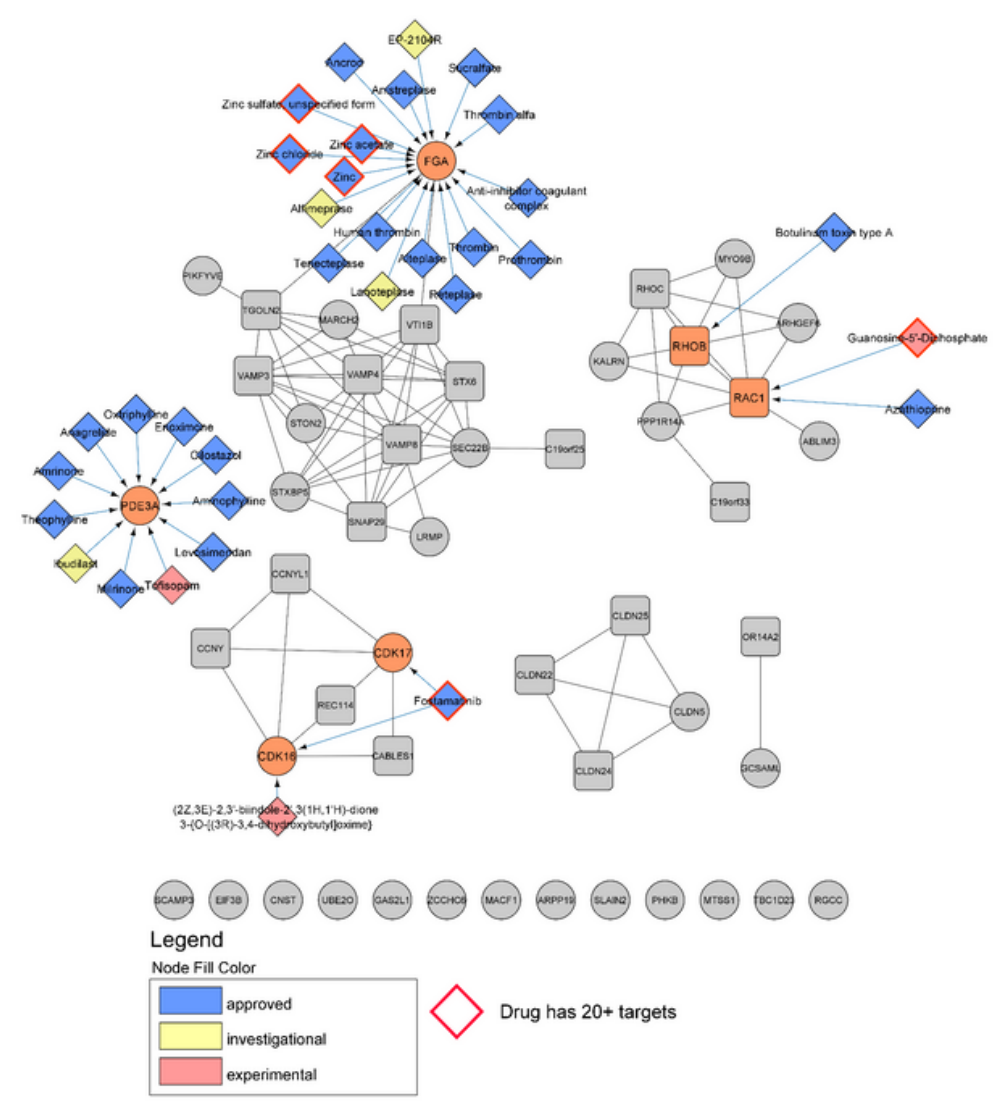

\section{Figure 6}

Protein-protein interaction network of potential "switch" candidates in platelet activation with FDAapproved drug target interactions from DrugBank. Drugs are represented by diamonds and targets are visualized as colored squares. PDE3A and FGA are targeted by many known drugs including generic ones such as zinc. Drugs that have more than 20 known targets are marked by a red outline. Node fill color represents the type of drug; blue = approved, yellow = investigational, red = experimental, orange $=$ drug target.

\section{Supplementary Files}

This is a list of supplementary files associated with this preprint. Click to download.

- supplementaltables.xlsx 